\title{
Radial Electron Collection in Dye-Sensitized Solar Cells
}

\author{
Alex B. F. Martinson, ${ }^{t, \ddagger}$ Jeffrey W. Elam, ${ }^{\ddagger}$ Jun Liu, ${ }^{\dagger}$ Michael J. Pellin, ${ }^{\dagger, \ddagger}$ \\ Tobin J. Marks, ${ }^{\dagger}$ and Joseph T. Hupp ${ }^{*} \dagger$ \\ Department of Chemistry and Argonne-Northwestern Solar Energy Research Center, \\ Northwestern University, 2145 Sheridan Road, Evanston, Illinois 60208, and Division \\ of Materials Science, Argonne National Laboratory, 9700 South Cass Avenue, \\ Argonne, Illinois 60439
}

Received May 28, 2008; Revised Manuscript Received July 24, 2008

\begin{abstract}
We introduce a new photoelectrode architecture consisting of concentric conducting and semiconducting nanotubes for use in dye-sensitized solar cells (DSSCs). Atomic layer deposition is employed to grow indium tin oxide (ITO) within a porous template and subsequently coat the high area photoelectrode with amorphous $\mathrm{TiO}_{2}$. Compared with control devices lacking a current collector within the pores, the new photoelectrode geometry exhibits dramatically higher current densities, an effect attributed to the radial collection of electrons.
\end{abstract}

In the most efficient DSSCs to date, the superior light harvesting efficiency of photons throughout the visible spectrum is realized by a $\sim 14 \mu \mathrm{m}$ thick nanoparticle photoanode with roughness $>2000 .{ }^{1}$ The implications of this architecture define DSSCs as a class of photovoltaics (PVs) with (1) an exceedingly large, heterogeneous interface through which electrons may be (parasitically) intercepted and with (2) slow electron transport controlled by trap-limited hopping through a relatively long and tortuous path to the transparent electrode. ${ }^{2,3}$

Given a charge collection time of milliseconds at the maximum power point, ${ }^{4}$ efficient charge extraction is only made possible through the extraordinarily slow interception of electrons by adjacent $\mathrm{I}_{3}{ }^{-}$. Thus, what promises to be a tour de force in photoelectrochemical energy conversion has so far only offered a limited variable space in which efficient devices are realized. Particularly lacking is an assortment of redox couples that permit reasonable collection efficiency, the current number able to be counted upon one hand. ${ }^{5-8}$ Furthermore, the most successful shuttle to date, iodide/ triiodide $\left(\mathrm{I}^{-} / \mathrm{I}_{3}{ }^{-}\right)$, builds in photovoltage losses of approximately 500 to $600 \mathrm{mV}$ on account of the high driving force needed to regenerate dyes at acceptable rates. Additionally, the iodine-based redox shuttle remains poorly understood owing to its multielectron nature and nonconformity to simple rate laws. ${ }^{9}$ One consequence of the "shuttle problem" has been efficiency improvements of only $\sim 1 \%$ in DSSC devices over the past 15 years. ${ }^{1,10}$

\footnotetext{
* To whom correspondence should be addressed. E-mail: j-hupp@ northwestern.edu.

$\dagger$ Northwestern University.

* Argonne National Laboratory.
}

In order to accommodate the faster electron interception rates of alternative redox shuttles, the collection time of electrons traversing the photoanode must be reduced. In the limit of rapid dye regeneration, the collection of electrons at the transparent conducting electrode (TCO) is in competition only with the interception of electrons by the oxidizing redox species. Since electron transport through the wide bandgap semiconductor is typically diffusion controlled, the transport time is inversely proportional to the effective electron diffusion constant and goes as the inverse square of photoanode thickness. Therefore, two distinct strategies may make devices more tolerant to faster interception (i.e., alternative redox couples), accelerating electron diffusion or reducing the photoanode thickness. Appeals to the first strategy are the motivation for recent reports of onedimensional (1D) nanorod and nanotube photoanodes with relatively large crystalline domains compared with nanoparticle photoanodes. ${ }^{11-13}$ Although yet to be tested with alternative redox couples, these novel photoanodes show potential to collect electrons over greater distances relative to their nanoparticle analogues. While the second strategy is straightforward, reducing the photoanode thickness results in a proportional reduction in surface area for dye loading, ultimately resulting in loss of light harvesting efficiency (unless suitable superchromophores are devised). Overviews of the intricacies of DSSC charge dynamics, as modeled by the continuity equation for DSSCs, have been communicated by several authors. ${ }^{14-17}$

Herein, we introduce a new device architecture in which the electron collection distance is reduced by orders of magnitude, without a significant reduction in surface area, 

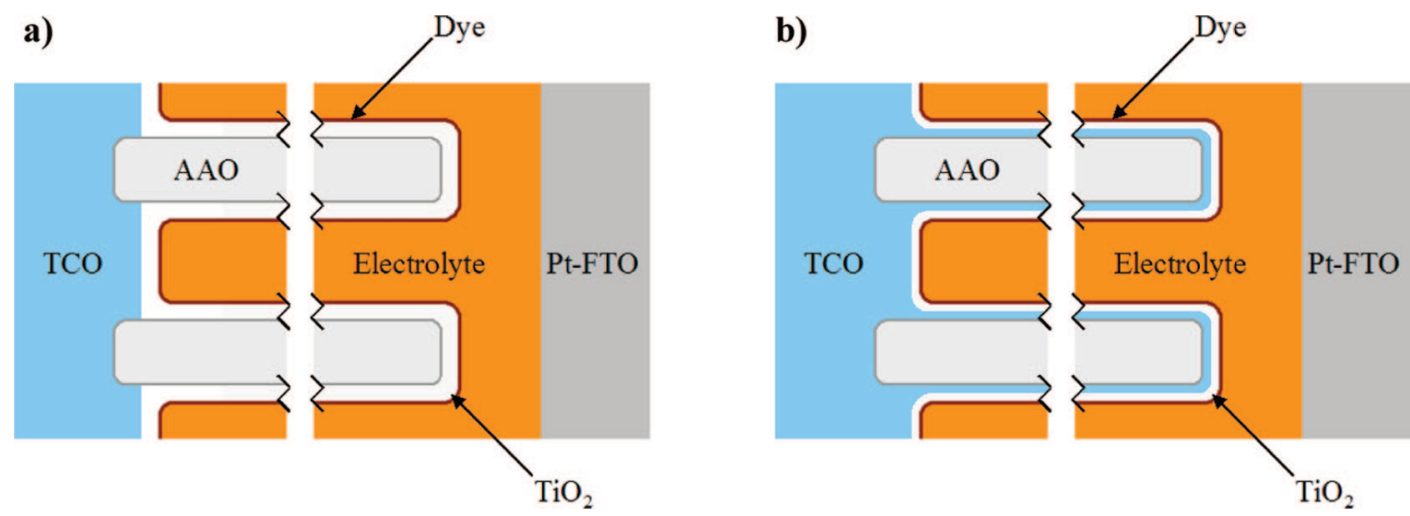

Figure 1. Idealized two-dimensional cross section of (a) a nanotube DSSC photoanode and (b) a concentric nanotube DSSC photoanode with ITO lining the pores.

by extending the transparent electrode deep within the semiconductor framework. The architecture is an elaboration of a previous design ${ }^{12}$ in which wide bandgap semiconductor tubes were grown within the pores of anodic aluminum oxide (AAO) by atomic layer deposition (ALD), Figure 1a. By leveraging the broad synthetic palette of ALD, a variety of metal oxides tubes may be fabricated inside AAO including transparent conducting oxides (TCOs). ${ }^{18-20}$ In this report, indium tin oxide (ITO) is grown within and upon AAO to produce a transmissive and conductive high-area electrode. Subsequent deposition of amorphous $\mathrm{TiO}_{2}$ within the pores of the electrode yields a high-area photoanode suitable for implementation in DSSCs. A highly idealized schematic of the new architecture is shown as Figure 1b. In contrast to a conventional DSSC photoanode, in which electrons diffuse the length of the micrometers-thick nanotube or nanoparticle photoanode, we hypothesized that electrons would flow radially through a few nanometers of semiconductor to be collected by the adjacent, interdigitated ITO electrode (iITO). While the idea of extending the current collector within a DSSC photoanode has been previously postulated, ${ }^{21}$ this work represents the first experimental realization. Furthermore, the materials and methods as well as nanoscale architecture reported herein are considerably different from those previously proposed.

In this report, we demonstrate the viability of the new photoelectrode geometry with a conventional redox couple $\left(\mathrm{I}^{-} / \mathrm{I}_{3}{ }^{-}\right)$by comparing its photovoltaic performance to that of control devices lacking ITO within the pores, Figure 1a. In many ways, the new nanoscale architecture is a return to simple bilayer heterojuction photovoltaics. The advantage here is that a large surface area (and correspondingly large dye-based light harvesting platform) can be packed into a small projected area by "folding" the heterojuction over a transparent template.

Materials and Methods. A nominally $60 \mu \mathrm{m}$ thick AAO membrane with $200 \mathrm{~nm}$ pores that is $25-50 \%$ porous (Anodisc 13, $0.02 \mu \mathrm{m}$, Whatman) was cleaned by $30 \mathrm{~s}$ sonication in water followed by an isopropanol rinse. The membranes were coated using atomic layer deposition (ALD) in a custom built viscous flow reactor ${ }^{22}$ operating under 1 torr of $\mathrm{N}_{2}$. For i-ITO samples, the membranes were coated with $\sim 7 \mathrm{~nm}$ of ITO by ALD via 45 alternate exposures to cyclopentadienyl indium ( $\mathrm{InCp}$ ) and $\mathrm{O}_{3}$ at a temperature of $220{ }^{\circ} \mathrm{C}$. Reactant exposure times of 15 and $80 \mathrm{~s}$ were employed, respectively, with nitrogen purge periods of 10 and $5 \mathrm{~s}$ after respective exposures. Doping of the $\mathrm{In}_{2} \mathrm{O}_{3}$ was accomplished by substituting pulses of tetrakisdimethylamido tin (TDMASn) and $\mathrm{H}_{2} \mathrm{O}_{2}$ every 10th cycle. The exposure times for TDMASn and $\mathrm{H}_{2} \mathrm{O}_{2}$ were $15 \mathrm{~s}$ and $10 \mathrm{~s}$, respectively, with $10 \mathrm{~s} \mathrm{~N}_{2}$ purge periods following each exposure. Postdeposition annealing of the membrane under $\mathrm{N}_{2}$ in a tube furnace at $450{ }^{\circ} \mathrm{C}$ for $20 \mathrm{~min}$ increased the conductivity of the i-ITO. A thick ( $230 \mathrm{~nm})$ ITO coating was applied to one side of both the i-ITO and the uncoated membranes by ion-assisted deposition (IAD) at room temperature. ${ }^{23}$ IAD is a line-of-sight deposition method similar to sputtering in which very little ITO penetrates the tapered $(20 \mathrm{~nm})$ pores of the asymmetric membrane. Finally, several different thicknesses of amorphous $\mathrm{TiO}_{2}$ were deposited within each membrane using ALD. During the $\mathrm{TiO}_{2}$ ALD, the membranes were mounted in a steel fixture designed to minimize $\mathrm{TiO}_{2}$ growth on the thick IAD ITO layer (to which electrical contact is subsequently made). The $\mathrm{TiO}_{2} \mathrm{ALD}$ was performed using alternating exposures to $\mathrm{TiCl}_{4}$ and $\mathrm{H}_{2} \mathrm{O}$ at $100{ }^{\circ} \mathrm{C}$ for 6 and 3 s respectively, with nitrogen purges of 10 and $14 \mathrm{~s}$ between exposures. Finally, each sample was soaked in an ethanoic solution of $0.5 \mathrm{mM}\left(\mathrm{Bu}_{4} \mathrm{~N}\right)_{2}\left[\mathrm{Ru}\left(4-(\mathrm{COOH}), 4^{\prime}-\right.\right.$ (COO)-2,2'-bipyridine $)_{2}(\mathrm{NCS})_{2}$ ] ("N719", Dyesol, B2 dye) for $20 \mathrm{~h}$ and rinsed with ethanol before devices were assembled according to established methods. ${ }^{1}$ Briefly, a 25 $\mu \mathrm{m}$ Surlyn spacer (Solaronix SX1170-25) was sandwiched between the photoanode and a platinized fluorine doped tin oxide (FTO) dark electrode. A $0.28 \mathrm{~cm}^{2}$ active area was defined by a hole punched through the spacer, which softens at $\sim 80{ }^{\circ} \mathrm{C}$ to seal the device. Electrical contact was made to each electrode by applying silver epoxy (SPI) to a tin-coated copper wire. A solution of $0.5 \mathrm{M}$ butylmethylimidizolium iodide (TCI America), $0.05 \mathrm{M} \mathrm{I}_{2}$, and $0.5 \mathrm{M}$ tert-butylpyridine in 85:15 acetonitrile/valeronitrile was introduced into the cell via vacuum backfilling through a hole in the platinized FTO electrode. A second Surlyn spacer and microscope coverslip were sealed over the hole with a soldering iron. All chemicals were used as received from Sigma-Aldrich unless otherwise specified. 
Monochromatic illumination for photoresponse experiments was achieved through the excitation slits of a JobinYvon fluorescence spectrometer. $J-V$ plots were acquired on a $\mathrm{CH}$ Instruments 1202 potentiostat. The AM 1.5 power efficiency of a $0.28 \mathrm{~cm}^{2}$ masked device was measured on a class A solar cell analyzer from Spectra-Nova Technologies. Scanning electron microscopy (SEM) was performed using a Hitachi S4800.

Results and Discussion. The viability of the i-ITO photoanode design relies upon the ability of ALD to coat the membrane pores conformally with transparent and conductive ITO. The development of ALD processes for $\mathrm{In}_{2} \mathrm{O}_{3}$ and $\mathrm{SnO}_{2}$ using nonhalogenated precursors make possible this challenging task. ${ }^{18-20}$ That the photoelectrodes employed herein are coated by a continuous film is demonstrated by resistivity measurements through the $60 \mu \mathrm{m}$ membranes. Though each nanoscale tube that spans a membrane is poorly conductive on its own, in parallel, the millions of tubes show a collective resistance $<20 \Omega$, in analogy to a conductive copper cable composed of many thin filaments. Subsequent annealing in $\mathrm{N}_{2}$ at $450{ }^{\circ} \mathrm{C}$ for 15 min reduces this value, measured by sandwiching the membrane between metal surfaces, to $<3 \Omega$.

To prevent the rapid (parasitic) reaction of electrons in the ITO with the hole transporting ions in solution $\left(\mathrm{I}_{3}{ }^{-}\right)$, an equally conformal semiconductor layer is required. To apply this semiconducting layer, $\mathrm{ALD}$ of $\mathrm{TiO}_{2}$ was performed using alternating exposures to $\mathrm{TiCl}_{4}$ and $\mathrm{H}_{2} \mathrm{O}$ at $100{ }^{\circ} \mathrm{C}$. This growth temperature was selected to minimize etching by $\mathrm{TiCl}_{4}{ }^{24}$ Micro-Raman analysis performed after the $\mathrm{TiO}_{2}$ ALD detected no anatase or rutile peaks on either the i-ITO or the bare alumina membranes (data not shown), implying that the semiconductor is generated in amorphous form as has been previously reported at this deposition temperature. ${ }^{25}$ Cross sectional SEM analysis of a fractured alumina membrane with i-ITO $(\sim 15 \mathrm{~nm})$ and $\mathrm{TiO}_{2}$ pore wall $(\sim 20$ $\mathrm{nm}$ ) reveals the bilayer structure of the photoanode, Figure 2. The smoothness of the $\mathrm{TiO}_{2}$ coating is further evidence for an amorphous film.

Figure 3 shows the short-circuit photocurrent densities $\left(J_{s c}\right)$ measured for a series of samples prepared with increasing $\mathrm{TiO}_{2}$ tube wall thickness, with and without i-ITO. The most salient observation is that all of the control samples, lacking i-ITO within the pores, exhibit very small current densities that only slowly increase with $\mathrm{TiO}_{2}$ thickness. The meager output of the control devices is not surprising since injected electrons, forced to traverse the micrometers-long amorphous $\mathrm{TiO}_{2}$ tubes, are expected to be collected extremely inefficiently at the distant TCO (see Figure 1a). We hypothesize that the diffusion/hopping of electrons through the amorphous semiconductor is too slow to compete effectively with their interception by $\mathrm{I}_{3}{ }^{-}$. The result is a short effective diffusion length over which injected electrons may be collected and consequent poor conversion efficiencies. These small $J_{s c}$ values are in contrast to the $J_{s c}$ of a similarly constructed $\mathrm{ZnO}$ nanotube $\mathrm{DSSC}^{12}$ in which the highly crystalline semiconductor provides an amply efficient electron transport pathway. An additional control, in which only i-ITO is

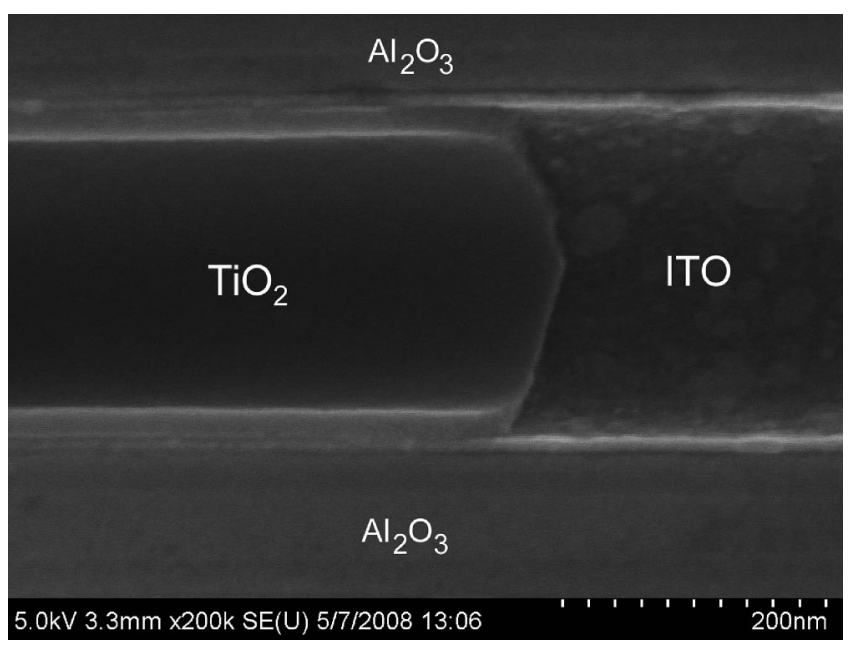

Figure 2. Cross-sectional SEM image of a $\mathrm{TiO}_{2}$ tube cleaved to reveal the i-ITO tube beneath. The concentric tubes, grown by atomic layer deposition, are embedded in a $\sim 60 \mu \mathrm{m}$ long alumina pore. In order to provide clear imaging, the $\mathrm{TiO}_{2}$ and i-ITO film thicknesses shown here are thicker than those utilized in photovoltaic devices.

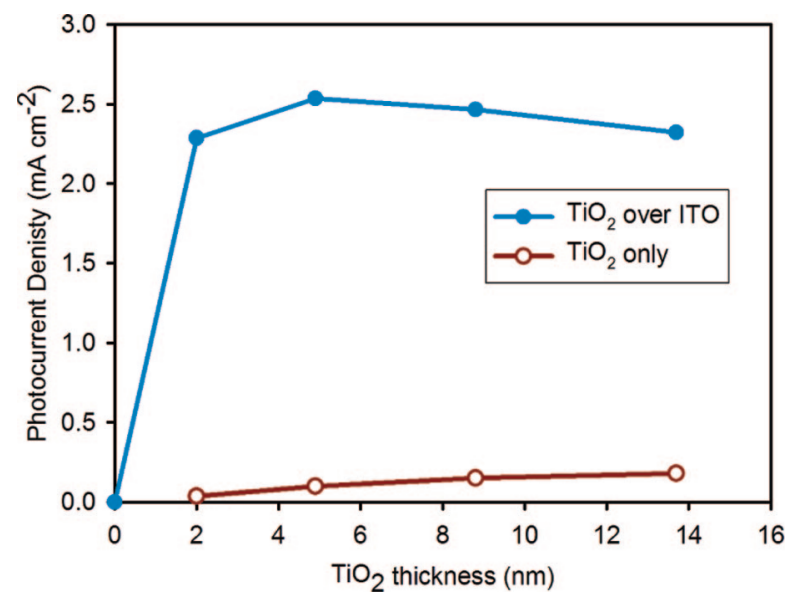

Figure 3. Short-circuit photocurrent density for devices with (blue, closed circle) and without (red, open circle) $7 \mathrm{~nm}$ of ITO in the pores as a function of $\mathrm{TiO}_{2}$ tube wall thickness.

present in the pores, underscores the need for some thickness of semiconductor to rectify the dark current. Here, the high surface area TCO exhibits exceedingly large dark currents that overwhelm any photocurrent. In striking contrast, the i-ITO DSSCs (idealized in Figure 1b), each with $>2 \mathrm{~nm}$ of $\mathrm{TiO}_{2}$ over $7 \mathrm{~nm}$ of ITO within the pores, show larger $J_{S C}$ values (10- to 60-fold) than the controls. Indeed, the current densities approach that of the best $\mathrm{ZnO}$ nanotube DSSCs to date. $^{12}$

In comparison with the best nanocrystalline DSSCs, however, nanotube $J_{S C}$ values are still low. Current densities of the nanotube devices are primarily limited by low light harvesting efficiency, a consequence of both the modest surface area of the AAO template $\left(\sim 400 \mathrm{~cm}^{2} / \mathrm{cm}^{2}\right)$ and the propensity of the commercial AAO samples to scatter incident photons and thereby inhibit light penetration. Both problems are, in principle at least, solvable by switching to custom-made, narrow-channel AAO templates. The extent 


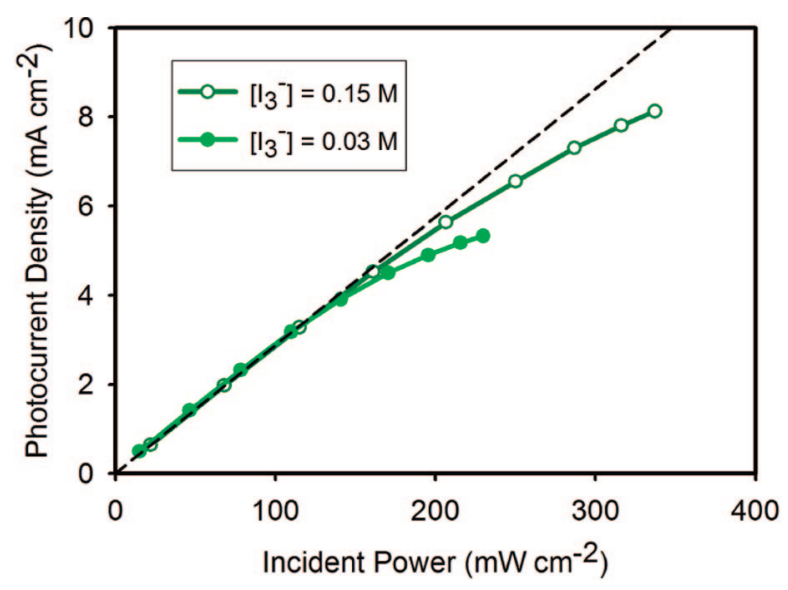

Figure 4. Short-circuit photocurrent density $\left(J_{S C}\right)$ of the most efficient i-ITO device reported (light green, closed circle) as a function of illumination intensity. The departure from linearity at high light intensity can be reduced by increasing the $\mathrm{I}_{3}{ }^{-}$to $0.15 \mathrm{M}$ (dark green, open circle) in an otherwise identical device. The dashed line defines a hypothetical linear $J_{S C}$ /incident-power correlation. Note that $100 \mathrm{~mW} / \mathrm{cm}^{2}$ corresponds approximately to 1 Sun of visible-region light intensity.

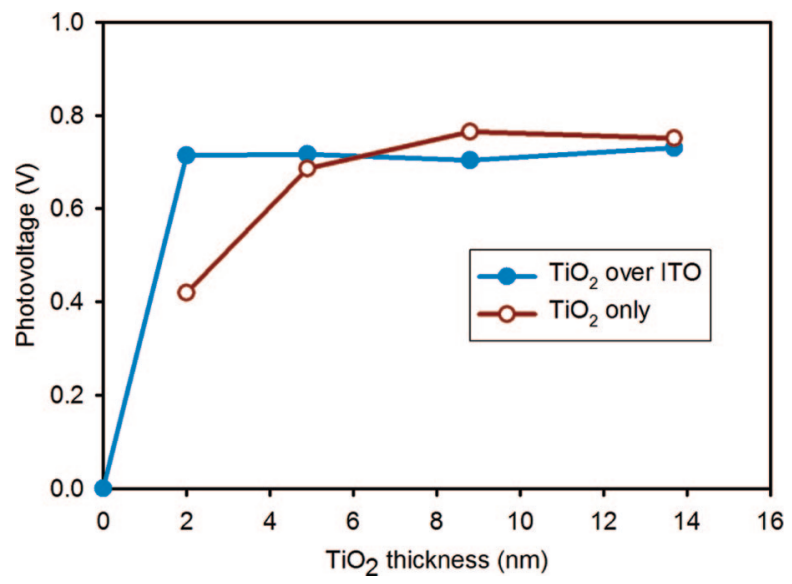

Figure 5. Open-circuit photovoltage for devices with (blue, closed circle) and without (red, open circle) ITO in the pores as a function of $\mathrm{TiO}_{2}$ tube wall thickness.

to which photocurrents are ultimately limited by ion transport through the liquid electrolyte is revealed by light intensity dependence measurements, Figure 4. The photocurrent increases linearly with light intensity, up to approximately $4 \mathrm{~mA} / \mathrm{cm}^{2}$. Beyond this point, mass transport of $\mathrm{I}_{3}{ }^{-}$through the relatively thick photoanode (among other possible issues) evidently becomes detrimental. This effect can be ameliorated to some extent by increasing the concentration of $\mathrm{I}_{3}{ }^{-}$in solution (see Figure 4).

The photovoltage at open circuit $\left(V_{O C}\right)$ of i-ITO devices is consistently greater than $700 \mathrm{mV}$, while the $J-V$ curve of the control device (without $\mathrm{TiO}_{2}$, but with i-ITO) passes through the origin. Apparently $\leq 2 \mathrm{~nm}$ of $\mathrm{TiO}_{2}$ is required to reach the maximum photovoltage for the system, Figure 5. Amorphous $\mathrm{TiO}_{2}$ tube DSSCs (without ITO in the pores) also exhibit good photovoltages that peak (or perhaps plateau) around $9 \mathrm{~nm}$. The $V_{O C}$ of the most efficient i-ITO DSSC reported here is within $80 \%$ of the $V_{O C}$ of that for the most efficient nanoparticle DSSCs to date $(850 \mathrm{mV})^{1}$ implying

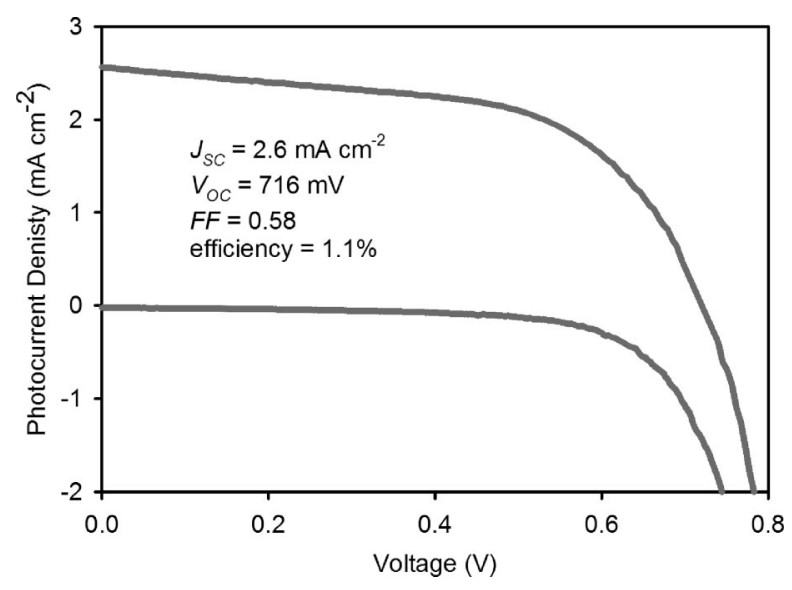

Figure 6. $J-V$ curve under AM 1.5 illumination and in the dark for the most efficient i-ITO device, $5 \mathrm{~nm} \mathrm{TiO}{ }_{2}$ over $7 \mathrm{~nm}$ ITO.

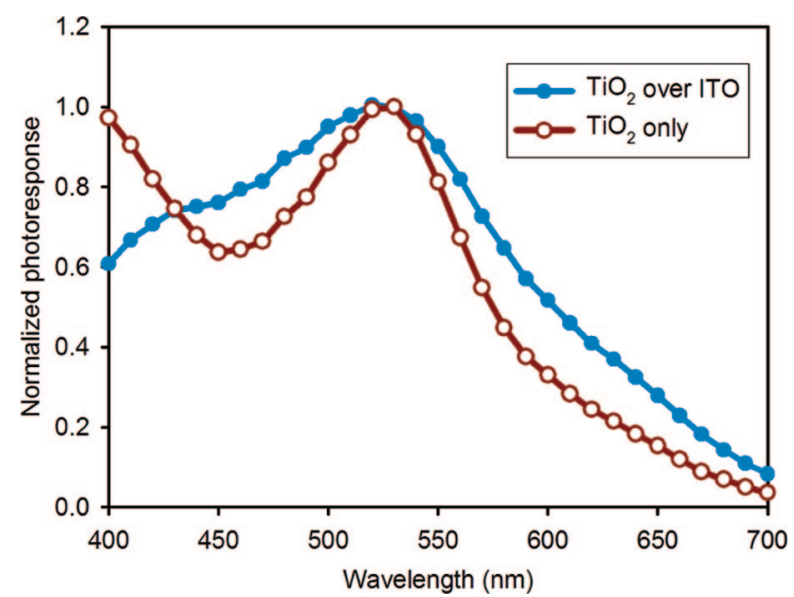

Figure 7. Representative normalized photoresponses under shortcircuit conditions for devices with (blue, closed circle) and without (red, open circle) ITO in the pores.

that the rectifying behavior of amorphous $\mathrm{TiO}_{2}$ is similar to that of anatase $\mathrm{TiO}_{2}$ (employed in nanoparticle cells).

The $J-V$ curve of the most efficient device, $5 \mathrm{~nm} \mathrm{TiO}_{2}$ over $7 \mathrm{~nm}$ ITO, is shown as Figure 6. Under AM 1.5 illumination, the DSSC exhibits a $J_{S C}$ of $2.6 \mathrm{~mA} / \mathrm{cm}^{2}, V_{O C}$ of $716 \mathrm{mV}$, and fill factor $(F F)$ of 0.58 , yielding an overall conversion efficiency of $1.1 \%$. The modest $F F$ is most likely due to a low shunt resistance, the signature of which can be seen in the negatively sloping photocurrent at low applied potentials. The origin of the shunt resistance is not yet clear.

Further insight into the operation of the new photoanodes can be gleaned from the spectral photoresponse of the completed devices. Because of the logarithmic relationship between absorbance and light harvesting efficiency, a longer path length cell will exhibit broadened features on a light harvesting efficiency scale. For example, the difference between a 1 and 2 absorbance unit valley and peak is small $(\sim 10 \%)$ on the light harvesting (1 minus transmission) scale. This effect can be seen in Figure 7 around the peak absorbance $(\sim 0.7)$ at $530 \mathrm{~nm}$. It appears that the path length (or in this case "useful" photoanode thickness as reported by photoresponse) is significantly shorter for devices without ITO in the pores. A deviation from this simple analysis is 
evident between wavelengths $400-430 \mathrm{~nm}$ where the i-ITO device has a smaller (normalized) photoresponse. This effect is most likely due to the attenuation of blue light traversing the cell by $\mathrm{I}_{3}{ }^{-}$and by ITO within the pores. The observation further supports the hypothesis that the useful photoanode thickness is greater for the DSSCs with ITO in the pores.

The substantially larger $J_{S C}$ and broadened photoresponse spectra of i-ITO DSSCs indicate that electrons are being collected over a much longer distance than in the control DSSCs. That is, radial electron collection through amorphous $\mathrm{TiO}_{2}$ (to surrounding ITO) competes effectively with electron interception, even deep within the photoelectrode. In contrast, longitudinal electron diffusion through amorphous $\mathrm{TiO}_{2}$ (to a distant planar ITO layer) does not. While the control devices based on amorphous $\mathrm{TiO}_{2}$ tubes were anticipated to perform poorly, the recovery of photovoltaic performance upon interdigitation of a TCO is remarkable and points to a broader utility for the radial collection approach. Particularly intriguing is the prospect of replacing traditional crystalline semiconductors (like anatase $\mathrm{TiO}_{2}$ ) with previously unexplored DSSC photoanode materials; amorphous $\mathrm{TiO}_{2}$ is now the first on this list. Since the task of rapid charge transport has been transferred to the i-ITO, effective use of otherwise inefficient materials such as electrodeposited and/or amorphous indirect bandgap semiconductors (including, perhaps, fuel producing photoelectrodes) may be feasible. Furthermore, the processing temperatures of DSSC photoanodes may be relaxed to allow for greater substrate compatibility.

In summary, a promising new DSSC photoanode design in which electrons radially diffuse through the wall of semiconducting tubes and are efficiently collected at an adjacent, concentric ITO tube has now been experimentally realized. Atomic layer deposition is used to grow the current collector within an AAO template and subsequently coat the high surface-area electrode with a semiconducting metal oxide. The viability of this architecture as a DSSC photoanode is demonstrated by photovoltaic characterization of completed devices with and without the interdigitated current collector. This architecture is not limited to AAO templates, and the benefits of radial charge collection may also be realized using other high surface area templates such as aerogels. ${ }^{26}$ Like previously reported nanotube array based DSSCs, the power efficiencies of these DSSCs are limited by modest light harvesting efficiency (at least with conventional dyes). Current work is focused on determining the extent to which optimized, higher-area templates can be translated into better photoelectrode performance and the extent to which the idea can be used to advantage with chemical producing (e.g., water oxidizing) photoelectrodes. Finally, these systems are good candidates for the exploration of alternative redox shuttles and previously unexplored semiconducting materials due to their potential for fast electron collection via the adjacent TCO.
Acknowledgment. The work at Northwestern was supported by the U.S. Department of Energy, Basic Energy Sciences Program, under Grant DE-FG02-87ER13808 and Grant DE-FG02-06ER46320. Work at Argonne was supported by the U.S. Department of Energy, BES-Materials Sciences under Contract W-31-109-ENG-38. SEM measurements were made in the EPIC facility of the NUANCE Center at Northwestern University. The NUANCE Center is supported by the NU NSF-NSEC, NU NSF-MRSEC, the Keck Foundation, the State of Illinois, and Northwestern University.

\section{References}

(1) Nazeeruddin, M. K.; De Angelis, F.; Fantacci, S.; Selloni, A.; Viscardi, G.; Liska, P.; Ito, S.; Takeru, B.; Gratzel, M. G. J. Am. Chem. Soc. 2005, 127 (48), 16835-16847.

(2) Dloczik, L.; Ileperuma, O.; Lauermann, I.; Peter, L. M.; Ponomarev, E. A.; Redmond, G.; Shaw, N. J.; Uhlendorf, I. J. Phys. Chem. B 1997, 101 (49), 10281-10289.

(3) van de Lagemaat, J.; Zhu, K.; Benkstein, K. D.; Frank, A. J. Inorg. Chim. Acta 2008, 361 (3), 620-626.

(4) Wang, Q.; Ito, S.; Gratzel, M.; Fabregat-Santiago, F.; Mora-Sero, I.; Bisquert, J.; Bessho, T.; Imai, H. J. Phys. Chem. B 2006, 110 (50), 25210-25221.

(5) Nusbaumer, H.; Moser, J. E.; Zakeeruddin, S. M.; Nazeeruddin, M. K.; Gratzel, M. J. Phys. Chem. B 2001, 105 (43), 10461-10464.

(6) Wang, Z. S.; Sayama, K.; Sugihara, H. J. Phys. Chem. B 2005, 109 (47), 22449-22455.

(7) Wang, P.; Zakeeruddin, S. M.; Moser, J. E.; Humphry-Baker, R.; Gratzel, M. J. Am. Chem. Soc. 2004, 126 (23), 7164-7165.

(8) Gorlov, M.; Pettersson, H.; Hagfeldt, A.; Kloo, L. Inorg. Chem. 2007, 46 (9), 3566-3575.

(9) Clifford, J. N.; Palomares, E.; Nazeeruddin, M. K.; Gratzel, M.; Durrant, J. R. J. Phys. Chem. C 2007, 111 (17), 6561-6567.

(10) Nazeeruddin, M. K.; Kay, A.; Rodicio, I.; Humphrybaker, R.; Muller, E.; Liska, P.; Vlachopoulos, N.; Gratzel, M. J. Am. Chem. Soc. 1993, 115 (14), 6382-6390.

(11) Law, M.; Greene, L. E.; Johnson, J. C.; Saykally, R.; Yang, P. D. Nat. Mater. 2005, 4 (6), 455-459.

(12) Martinson, A. B. F.; Elam, J. W.; Hupp, J. T.; Pellin, M. J. Nano Lett. 2007, 7 (8), 2183-2187.

(13) Mor, G. K.; Shankar, K.; Paulose, M.; Varghese, O. K.; Grimes, C. A. Nano Lett. 2006, 6 (2), 215-218.

(14) Sodergren, S.; Hagfeldt, A.; Olsson, J.; Lindquist, S. E. J. Phys. Chem. 1994, 98 (21), 5552-5556.

(15) Peter, L. M. J. Phys. Chem. C 2007, 111 (18), 6601-6612.

(16) Lee, J. J.; Coia, G. M.; Lewis, N. S. J. Phys. Chem. B 2004, 108 (17), 5282-5293.

(17) Martinson, A. B. F.; Hamann, T. W.; Pellin, M. J.; Hupp, J. T. Chem.Eur. J. 2008, 14 (15), 4458-4467.

(18) Elam, J. W.; Baker, D. A.; Martinson, A. B. F.; Pellin, M. J.; Hupp, J. T. J. Phys. Chem. C 2008, 112 (6), 1938-1945.

(19) Elam, J. W.; Martinson, A. B. F.; Pellin, M. J.; Hupp, J. T. Chem. Mater. 2006, 18 (15), 3571-3578.

(20) Elam, J. W.; Baker, D. A.; Hryn, A. J.; Martinson, A. B. F.; Pellin, M. J.; Hupp, J. T. J. Vac. Sci. Technol., A 2008, 26 (2), 244-252.

(21) Chappel, S.; Grinis, L.; Ofir, A.; Zaban, A. J. Phys. Chem. B 2005, 109 (5), 1643-1647.

(22) Elam, J. W.; Groner, M. D.; George, S. M. Rev. Sci. Instrum. 2002, 73 (8), 2981-2987.

(23) Yang, Y.; Huang, Q. L.; Metz, A. W.; Ni, J.; Jin, S.; Marks, T. J.; Madsen, M. E.; DiVenere, A.; Ho, S. T. Adv. Mater. 2004, 164, 321+.

(24) Puurunen, R. L. Chem. Vap. Deposition 2005, 11 (2), 79-90.

(25) Aarik, J.; Aidla, A.; Uustare, T.; Sammelselg, V. J. Cryst. Growth 1995, 148 (3), 268-275.

(26) Hamann, T. W.; Martinson, A. B. F.; Elam, J. W.; Pellin, M. J.; Hupp, J. T. Adv. Mater. 2008, 20 (8), 1560-1564.

NL8015285 